Search Alerts My list Settings

Back to results | 1 of $\mathbf{1}$

View at Publisher | Journal Finder | Library Catalogue | \$ \$ WebBridge | Export | Download | More...

Proceedings of SPIE - The International Society for Optical Engineering

Cite

Volume 7613,2010 , Article number 761300

Complex Light and Optical Forces IV; San Francisco, CA; United States; 27 January 2010 through 28

January 2010; Code 79522

\title{
Collinear non-diffracting beams: Classification and properties
}

(Conference Paper)

Ahluwalia, B.S. ${ }^{a} \quad$, Lee, W.M. $^{\text {b }}$

a Department of Physics and Technology, University of Tromsø, Tromsø 9037, Norway

b SUPA, School of Physics and Astronomy, University of St. Andrews, St Andrews, Fife KY16 9SS, United Kingdom

Relı́

\section{Abstract}

View references (32)

Non-diffracting laser modes and interfering non-diffracting beams have been extensively studied. Interfering nondiffracting beams generate novel laser modes. In this paper we accumulate various interfering conditions for nondiffracting beams and discuss the properties of the resultant beam. Contrasting intensity profiles and topological charge distribution are obtained on varying interfering conditions. Collinear propagation of non-diffracting laser beams is also reported. (c) 2010 Copyright SPIE - The International Society for Optical Engineering.

\section{Author keywords}

Bessel beams; Collinear Bessel beams; Helicon beams; Non-diffracting beams

\section{Indexed keywords}

Bessel beam; Intensity profiles; Non-diffracting beams; Nondiffracting; Nondiffracting beam; Topological charges

Engineering controlled terms: Bessel functions; Helicons; Laser modes

Engineering main heading: Laser beams

ISSN: 0277786X ISBN: 978-081948009-5 CODEN: PSISD Source Type: Conference Proceeding Original language: English

DOI: 10.1117/12.840390 Document Type: Conference Paper

Sponsors: The Society of Photo-Optical Instrumentation Engineers(SPIE)

References (32) 